\title{
De l'évolution des problèmes relatifs à l'hygiène du lait (1)
}

\author{
par \\ James H. STEELE, DVM, MPH (2) \\ et Carl D. OLSEN, DVM, MPH (3)
}

Il est assez piquant de constater que, capables d'envoyer des hommes graviter autour de la lune, nous ne sommes pas encore a l'abri des maladies transmises par le lait. Les résultats obtenus dans le domaine interplanétaire sont le fruit des recherches intensives de ces vingt dernières années, alors que les savants depuis des siècles se penchent sur le problème des maladies transmises par le lait. Certes, la tuberculose et la brucellose bovines ont presque disparu. De nos jours les effets des maladies transmises par le lait sont infimes si on les compare à ceux enregistrés il y a un demi-siècle. Cependant les responsables des Services de la Santé publique sont confrontés dans ce domaine à bien des difficultés. Les techniques laitières ont évolué rapidement et de nouveaux problèmes apparaissent qu'il faut résoudre afin d'assurer une production toujours croissante de lait propre provenant de vaches saines.

Jusqu'à une époque très récente le Service de la Santé publique des U.S.A. admettait deux rapports " temps/température » correspondant aux normes fixées pour la pasteurisation du lait. En raison des viscosités variables des différents produits laitiers, les temps de chambrage dans les tubes des appareils H T S T étaient fonction du produit à pasteuriser. Par injection directe de vapeur dans le lait, on a pu presque instantanément dépasser les $71,7^{\circ} \mathrm{C}\left(161^{\circ} \mathrm{F}\right)$. A cette température plus élevée, le temps de séjour dans les tubes de chambrage a pu être considérablement réduit, mais en raison des viscosités variables des différents produits laitiers, le temps de séjour fut malaisé à déterminer. En outre, l'écoulement laminaire

(1) Rapport présenté au $5^{\text {me }}$ Symposium W.A.V.F.H., Opatïja (Yougoslavie), 22-27 septembre 1969.

(2) Assistant Surgeon Général. Nat. Com. Dis. C., U.S.P.H.S. D.H.E.W., Atlanta, Georgia, 30333 (U.S.A.).

(3) Surveillance and Intelligence officier, Division of Food, Milk and Intern. Tr. Sanit. E.C.A., U.S.P.H.S., D.H.E.W., Cincinnati, 45202 (U.S.A.). 
rendait nécessaire la détermination du temps de séjour minimum des particules les plus rapides. Après des recherches attentives, par l'utilisation d'instruments ultra-modernes tels que " thermistors " et chronomètres électroniques, la Section d'Etudes laitières du Service d'hygiène publique des U.S.A. a mis au point une formule qui permet de calculer exactement la longueur du tube de chambrage en accord avec les normes de pasteurisation. Le Service d'hygiène publique admet maintenant pour le lait et les produits laitiers la méthode de pasteurisation UHT. On a défini le système UHT comme un ensemble de processus thermiques employés pour les produits laitiers dont le temps de chambrage ne dépasse pas 2 s, et dont la température de chambrage oscille entre $87,8^{\circ} \mathrm{C}$ et $132,2^{\circ} \mathrm{C}$ $\left(190^{\circ}\right.$ et $\left.270^{\circ} \mathrm{F}\right)$. On a retenu la limite supérieure attendu que la plupart des opérations à des températures plus élevées concernent la stérilisation, et non la pasteurisation. Les exemples de pasteurisation U H avec le rapport "temps/température », admissible, sont les suivants :

\begin{tabular}{|c|c|c|c|c|}
\hline $90,0^{\circ} \mathrm{C}\left(194^{\circ} \mathrm{F}\right)$ & 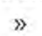 & » & 27 & de 0,5 \\
\hline $94,0^{\circ} \mathrm{C}\left(201^{\circ} \mathrm{F}\right)$ & $»$ & $»$ & $\gg$ & de 0,1 \\
\hline $95,5^{\circ} \mathrm{C}\left(204^{\circ} \mathrm{F}\right)$ & $»$ & » & $》$ & de $0,05 \mathrm{~s}$ \\
\hline $100,0^{\circ} \mathrm{C}\left(212^{\circ} \mathrm{F}\right)$ & $»$ & $»$ & 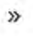 & de 0,01 \\
\hline
\end{tabular}

Le Service de la Santé publique a publié un fascicule qui détaille les procédés destinés à mesurer le temps de chambrage et la vitesse de réponse du système de contrôle [15].

Bien que les effets des maladies transmises par le lait soient faibles, les responsables de la Santé publique ne doivent pas devenir présomptueux. On accorde trop de confiance à la pasteurisation effective du lait, ainsi qu'à la réfrigération pour ralentir le développement des bactéries, et au coupage d'un lait peut-être suspect avec le lait de qualité provenant d'une autre source. Les services de contrôle du lait savent le risque encouru si d'aventure divers facteurs venaient à être réunis. "Ces facteurs pourraient être la présence simultanée d'infections par staphylocoques dans plusieurs troupeaux d'une même entreprise, ou une panne de réfrigération ", ayant pour résultat une production de quantités importantes d'entérotoxines staphylococciques. Les temps et températures-couramment employés pour la pasteurisation du lait sont inefficaces quand il s'agit de neutraliser les entérotoxines staphylococciques. A $98,9^{\circ} \mathrm{C}$ $\left(210^{\circ} \mathrm{F}\right)$ le lait doit être maintenu pendant $68,5 \mathrm{mn}$ pour neutraliser 90 p. 100 d'entérotoxines pures.

Six ans après que furent écrites ces lignes prémonitoires, un concours de circonstances eut pour effet environ 147 cas d'intoxication par staphylocoques après consommation de lait pasteurisé. Une petite entreprise de pasteurisation traitait environ 26351 de lait par jour (700 gallons), provenant de deux fournisseurs. Par suite d'un accord spécial, on traita en plus 22711 (600 gallons) d'un troi- 
sième producteur. Ce supplément fut traité séparément et stocké dans des containers étiquetés spécialement sous le nom de ce troisième producteur. Or, les consommateurs de ce lot supplémentaire furent touchés, et non pas les clients habituels de la laiterie considérée. La numération microbienne du lait incriminé fut faible et le test à la phosphatase montra que le lait avait été convenablement pasteurisé. La numération directe au microscope, cependant, révéla de grandes quantités d'organismes présumés non viables. Des échantillons de lait cru furent alors prélevés chez le fournisseur suspecté et indiquèrent la présence de staphylocoques à coagulase positive à un taux inférieur à 300 unités par ml. Les enquêteurs découvrirent qu'on avait négligé de mettre en marche le système de réfrigération de la citerne lors de la traite du soir, et que le lait était demeuré à la température ambiante toute la nuit.

Le lait de consommation n'est pas seul en cause. Ainsi, un important complexe fromager du Midwest se vit saisir $1846152 \mathrm{~kg}$ de fromages parce qu'on y soupçonnait la présence d'entérotoxines staphylococciques. Pour permettre la levée de la saisie chaque lot fut analysé pour rechercher l'entérotoxine staphylococcique A par la technique de diffusion en plaques minces de gélatine. Sur les 2112 lots analysés, 59 (2,79 p. 100) se révélèrent toxiques [23].

On n'a pas enregistré d'infections dues aux salmonelles provenant de lait correctement pasteurisé aux U.S.A. ; cependant on reçoit toujours des rapports concernant quelques cas d'intoxication provenant de la vente de lait cru. Heureusement, on estime que plus de 99 p. 100 du lait consommé en nature aux U.S.A. est pasteurisé. Des cas d'infection pouvant être attribués au lait écrémé instantané étaient en fait dus à une pasteurisation incorrecte ou à une contamination intervenant après la pasteurisation. Depuis ces deux dernières années, presque tous les lots de ce produit sont analysés en laboratoire pour rechercher les salmonelles. Au cours d'une enquête à la suite d'un début d'épidémie, l'épidémiologiste doit se garder de mettre hors de cause un lait ou un produit laitier suspects en se fondant seulement sur les résultats négatifs des tests suivant la pasteurisation. Il est évident que certaines bactéries sont apparemment touchées par la chaleur lors de la pasteurisation. Quand le produit considéré est ensemencé sur plaque de gélose à l'issue de la pasteurisation, il se produit un ralentissement pendant lequel aucune culture n'apparaît avant le troisième ou quatrième jour [14]. Les plaques lues et mises hors de cause après $48 \mathrm{~h}$ peuvent être à tort considérées comme sans culture.

Les chercheurs de la Section d'Hygiène du lait de Cincinnati travaillent sur deux groupes de pathogènes possibles du lait qui n'ont guère attiré l'attention des responsables de l'hygiène du lait jusquà ces tous derniers temps.

Les premiers de ces dangers latents sont les virus et les organismes similaires. La découverte de mammite à mycoplasme dans le 
bétail n'est pas nouvelle mais elle est signalée assez souvent. Récemment, 10 vaches d'un lot de 80 vaches Holstein furent atteintes de mammite dans les 4 quartiers. Au laboratoire on isola un mycoplasme (P P L O) du lait de 4 vaches [16]. Les Auteurs n'indiquèrent pas si cette souche particulière était pathogène pour l'homme, mais l'un de nos ouvrages de référence, citant les travaux de I. Alström, note que certaines souches de mycoplasme résistent à la pasteurisation [3]. Outre le mycoplasme, on indique avoir isolé différents virus (parainfluenza), myxovirus, pox virus, virus de l'herpès. Le laboratoire d'hygiène du lait du service de la Santé publique de Cincinnati est parvenu à isoler à la fois les virus de polio 1 et 3 à partir d'échantillons de lait cru et de lait cru de mammite.

En second lieu, un domaine riche en découvertes serait celui des mycobactéries atypiques ou non classées du lait. Ce problème fut porté à l'attention du Service d'Hygiène publique quand on vint à signaler que, sur 770 échantillons de lait cru provenant de wagonsciternes, 33,9 p. 100 contenaient des mycobactéries [1]. Le Service de la Santé publique fut à même de découvrir des mycobactéries dans 75 des 126 échantillons de lait cru prélevés à proximité du laboratoire de recherches. D'autres travaux signalèrent 83 souches différentes de mycobactéries provenant de 77 échantillons de lait cru sur 123. Beaucoup de ces souches étaient semblables aux souches pathogènes pour l'animal et l'homme [10].

Dans une étude précoce sur la résistance à la chaleur (1961), 30 souches de mycobactéries achromogènes furent soumises à un test de survie à $60^{\circ} \mathrm{C}$. L'ensemble comprenait 10 souches isolées de volailles, 10 des porcs et 10 d'hommes. De ces 30 souches, 27 survécurent à $60^{\circ} \mathrm{C}$ pendant $30 \mathrm{mn}$ et 4 pendant $2 \mathrm{~h}$ [19]. D'autres recherches effectuées dans le temps et à la température requis pour la pasteurisation rapide à haute température $\left(71,7^{\circ} \mathrm{C} / 15 \mathrm{~s}\right)$ donnèrent des résultats voisins. Le test fut effectué sur du lait écrémé, du lait non entier, mais sur les 161 souches de mycobactéries non classées et soumises au test, 11 survécurent en petit nombre [9].

L'épidémiologie des infections dues aux mycobactéries non classées chez l'homme est très curieuse. Le schéma d'ensemble diffère grandement de celui de l'infection tuberculeuse (Myc. tuberculosis) chez l'homme. Dans plusieurs études complétées ces dernières années, on ne mentionna nul cas d'extension familiale d'infections dues à des mycobactéries non classées. Au Texas la lymphadémie due à des mycobactéries non classées chez les enfants est surtout répandues à l'issue des deux mois qui suivent la période d'isolement la plus complète des organismes provenant de lait cru [1]. Dans une étude portant sur 2173 cas en Floride, l'infection a été plus fréquente chez l'homme de race blanche que chez la femme dans une proportion de 3 p. 1 . Chez les gens de couleur, le rapport est de 2 p. 1 . Les habitants des campagnes semblent être plus souvent atteints que les habitants des villes [4]. 
Dans une étude réalisée à Chicago sur le groupe 1 des mycobactéries non classées, le rapport était de 159 hommes de race blanche pour 26 femmes, et chez les gens de couleur de 30 p. 15. La contamination était plus fréquente parmi les hommes de race blanche disposant de revenus moyens [12].

Un autre chercheur a identifié 692 types différents de mycobactéries non classées par sérotypes. Les cultures furent obtenues à partir d'expectorations humaines, de lymphes de nodosités cervicales chez l'enfant, l'oiseau, le porc et le bétail. Des 18 sérotypes isolés sur l'homme, 14 furent retrouvés également sur les oiseaux ou autres animaux [20].

Tout programme se fixant pour tâche de combattre des infections transmises par le lait se doit de commencer par le commencement : le pis de la vache. Le Service de la Santé publique des U.S.A. est heureux de signaler que, grâce à la coopération de responsables locaux et nationaux de l'hygiène du lait, les Etats-Unis ont à leur disposition un programme national pour le contrôle du lait anormal [8]. Malgré l'apparition des antibiotiques, la fréquence de la mammite bovine aux U.S.A. n'a fait que croître. Une université réalisa une étude sur les pathogènes possibles pour l'homme dans le pis de la vache, en liaison avec différentes réactions au test de la mammite (Californian test). Les non pathogènes étaient plus nombreux dans les quartiers révélant une trace négative ou une réaction +1 . Chez ceux qui avaient une réaction $+2,64,7$ p. 100 des souches isolées étaient de possibles pathogènes pour l'homme ; dans les quartiers ayant une réaction +3 , la proportion s'élevait à 71,3 p. 100 [22].

En raison de l'étendue du programme de contrôle du lait anormal du Service de la Santé publique américain, le programme fut réalisé en trois temps successifs. D'abord, une période d'organisation. Les responsables locaux de l'hygiène du lait furent requis de réaliser un minimum de quatre tests tous les six mois en vue de la numération des cellules somatiques, sur un échantillon mixte de chaque troupeau. Ils furent également conviés à organiser des comités locaux ainsi qu'au niveau des Etats pour aider les fermiers laitiers dans la lutte contre la mammite. De tels comités comprenaient des vétérinaires, des responsables officiels de l'hygiène du lait, des inspecteurs du contrôle de la qualité dans les laiteries, des utilisateurs de machines à traire, des professeurs d'agriculture et des industriels laitiers. En résumé, furent admis tous ceux qui étaient désireux et capables de contribuer à résoudre le problème. Les épreuves de triage rapide déterminèrent l'importance du problème et tracèrent une ligne de base en fonction de laquelle on précisa les modalités de l'efficacité future du programme.

Pendant la deuxième phase, en place dès le $1^{\text {er }}$ juillet 1968 , des correctifs furent rendus nécessaires lorsque trois des quatre derniers tests dépassèrent 1500000 cellules somatiques par ml. Ceci peut 
comprendre l'appréciation et la correction d'erreurs techniques de traite, la correction d'équipements mécaniques défectueux, et/ou le secours d'un vétérinaire laitier. Une réduction surprenante de la fréquence de la mammite put être obtenue en corrigeant les mauvaises manipulations et les défauts d'équipement. Contrairement à l'opinion couramment répandue, on ne naît pas industriel laitier, on le devient.

Il est prévu qu'une troisième phase, phase de stricte application, débutera le $1^{\text {er }}$ juillet 1970 . A cette époque, les fournisseurs laitiers dont le lait dépassera 1500000 cellules somatiques par $\mathrm{ml}$ sur trois des quatre derniers tests n'auront plus le droit de vendre leur lait. L'autorisation ne leur sera pas redonnée tant que n'aura pas été supprimée la cause provoquant ce nombre élevé de cellules. Certaines régions de notre territoire sont en avance sur le programme et vivent cette troisième étape depuis plusieurs années. Certaines ont un maximum de 1000000 de cellules somatiques par $\mathrm{ml}$, et une région, Spokane (Washington), a un maximum de 800000 cellules somatiques par ml.

Pour ceux d'entre vous qui n'ont pas un programme de cet ordre, un mot d'avertissement. Les prophètes de malheur s'opposeront toujours à vous. Les raisons pour ne rien faire sont nombreuses, nous les avons entendues. A ceux qui désirent une liste complète de ces raisons pour ne rien faire, nous pouvons en fournir une. Malgré les prophéties menaçantes de certains, les industriels laitiers de Spokane (Washington) reconnaissent volontiers que le coût des médications pour le traitement de la mammite a baissé et que la production de lait par tête de bétail a augmenté après que la phase d'application du programme fut entrée en vigueur depuis plusieurs années. Au Congrès de 1969 du Conseil national de la mammite, un industriel laitier du sud-est a déclaré avoir réduit ses dépenses dans la lutte contre la mammite jusqu'à 10 p. 100 du coût initial.

Le programme de contrôle du lait anormal a stimulé quelques recherches bénéfiques. Un Comité de plusieurs chercheurs bien connus a modifié la technique de Breed de numération des leucocytes. Reconnaissant que toutes les cellules à noyau dans les frottis ne sont pas des leucocytes, le nom avancé pour la méthode de Breed amendée est la numération microscopique directe des cellules somatiques [21]. En utilisant un réticule spécial ce sont les bandes qui traversent la pellicule entière qui sont comptées plutôt que les champs séparés. Une plus grande précision de numération et de reproductibilité des résultats est signalée par cette nouvelle méthode de numération. Pour les laboratoires les plus importants on peut employer un compteur électronique. L'exactitude et la reproductibilité du compteur électronique dépasse de loin n'importe lequel des anciens tests pour déterminer la proportion de cellules dans le lait cru. 
En sus de la qualité du lait cru en vue de la pasteurisation, l'accent est mis tout particulièrement sur l'amélioration de la conservabilité à température ambiante du produit fini. Le plan de livraison du lait à domicile est passé d'une fois par jour à trois puis deux fois par semaine. Bien des ménagères achètent leur lait liquide pour une semaine lors de leur montée hebdomadaire au super-marché. Dans une étude effectuée sur des échantillons de marché pris au hasard dans des épiceries, dix marques différentes de lait " half and half " - à 10,5 p. 100 de matière grasse et 10,5 p. 100 d'extrait sec dégraissé - furent étudiés. La conservabilité moyenne depuis le moment de l'emballage était de $12,2 \mathrm{j}$, les extrêmes étant de 7,9 j et $17,5 \mathrm{j}$ [11]. Une seconde étude concerna l'effet de la température de stockage sur la conservabilité escomptée. Quand un lait entier pasteurisé commercialisé $\left(76,1^{\circ} \mathrm{C} / 16 \mathrm{~s}\right)$ provenant de neuf entreprises différentes était stocké à $7,3^{\circ} \mathrm{C}, 15 \mathrm{p}$. 100 seulement des échantillons étaient satisfaisants plus de $7 \mathrm{j}$. Mais lorsque d'autres échantillons de ces mêmes lots étaient stockés à $0^{\circ} \mathrm{C}, 81$ p. 100 des échantillons étaient acceptables pendant 3 semaines ou plus. Quand le lait était soumis aux méthodes $\mathrm{UH} \mathrm{T}$, à $104,4^{\circ} \mathrm{C}$ et stocké à $0^{\circ} \mathrm{C}$, la conservabilité dépassait 15 semaines. A ce moment, les échantillons furent livrés et la date limite ne put être déterminée [5].

La pasteurisation à très haute température et en temps réduit est de plus en plus en faveur. Le produit fini à une saveur meilleure et présente presque les caractéristiques de la stérilisation [13]. Si ce produit est conservé dans un système fermé, rempli aseptiquement dans des containers préalablement stérilisés, le lait liquide peut se garder environ 30 à $60 \mathrm{j}$ sans réfrigération.

La vente de l'oléomargarine est maintenant supérieure à celle du beurre de crème aux U.S.A., mais le grand succès de ces dernières années sont les produits artificiels pour "blanchir " le café. Ces derniers ont conquis une grande partie du marché. On vend à présent du lait artificiel tiré d'huiles et de protéines végétales. Bien que l'on doive améliorer sa saveur, son bas prix le rend commercialisable.

Le fromage blanc est conservé par lyophilisation et expédié à nos troupes d'outre-mer, mais ce procédé est encore trop coûteux pour en permettre une large diffusion en vue de la consommation courante [6].

Les Responsables de l'hygiène du lait ont résolu d'anciens problèmes, mais de nouveaux surgissent. La présence possible d'aflatoxines dans le fromage, de virus voisins de ceux de la leucémie dans le lait, les mycobactéries non classées, le lait anormal, et la nécessité d'une conservabilité plus longue : tels sont les problèmes à résoudre dans un avenir proche. Sinon, le lait artificiel a fortes chances de renouveler l'histoire de l'oléomargarine.

(Traduction Jean THIEULIN). 